In press at Canadian Psychology.

(c)American Psychological Association, 2017

\title{
Statcheck in Canada: What Proportion of CPA Journal Articles Contain Errors in the Reporting of $p$-Values?
}

\footnotetext{
Christopher D. Green, Sahir Abbas, Arlie Belliveau, Nataly Beribisky, Ian J. Davidson, Julian DiGiovanni, Crystal Heidari, Shane M. Martin, Eric Oosenbrug, \& Linda M.

Wainewright

York University
}

\author{
Contact: \\ Christopher D. Green \\ Department of Psychology \\ York University \\ Toronto, ON M3J 1P3 \\ Canada \\ e-mail:christo@yorku.ca
}

Keywords: Canada, journals, psychology, p-values, statistics 
Statcheck in Canada: What Proportion of CPA Journal Articles

Contain Errors in the Reporting of $p$-Values?

\begin{abstract}
Using a computer program called "Statcheck," a 2016 digital survey of several prestigious American and European psychology journals showed that the $p$-values reported in research articles failed to agree with the corresponding test statistics (e.g., $F, t, \chi^{2}$ ) at surprisingly high rates: nearly half of all articles contained at least one such error, as did about $10 \%$ of all null hypothesis significance tests. We investigated whether this problem was present in Canadian psychology journals and, if so, at what frequency. We discovered similar rates of $p$-value errors in Canadian journals over the past 30 years. However, we also noticed, a large number of typographical errors in the electronic versions of the articles. When we hand corrected a sample of our articles, the per-article error rate remained about the same, but the per test rate of errors dropped to $6.3 \%$. We recommend that, in future, journals include explicit checks of statistics in their editorial processes.
\end{abstract}




\section{Statcheck in Canada: What Proportion of CPA Journal Articles Contain Errors in the Reporting of $p$-Values?}

Psychology is currently undergoing a period of intense self-examination in light of a number of recent, disappointing discoveries. Seemingly well-established phenomena have been shown not to replicate reliably (Open Science Collaboration, 2015). Important questions have been raised about the integrity with which the standard statistical practices of the field are carried out (John, Loewenstein, \& Prelec, 2012; Masicampo \& Lalande, 2012; Simmons, Nelson, \& Simonsohn, 2011). One researcher has even enumerated a set of "diseases" with which behavioral scientific research is currently best (Antonakis, 2017). Most spectacularly, there have been a few highly visible cases of outright research fraud (e.g., Carpenter, 2012; Enserink, 2012). Matters such as these not only raise questions among psychologists. They also lead people and institutions outside of the discipline to publicly wonder whether psychological findings can be trusted in general (e.g., Carroll, 2017; Kessler, 2017; Yong, 2015).

Virtually all working psychologists have been trained in statistical analysis since they were undergraduate students, and the Null Hypothesis Significance Test (NHST) with its $p$-value has been the workhorse of the field since the mid- $20^{\text {th }}$ century (Gigerenzer, 2004). There is a long history of critique of the practice, both vociferous and moderate (e.g., Bakan, 1967; Cohen, 1962, 1992, 1994; Lykken, 1968; Meehl, 1967, 1978; Nunnally, 1960; Rosenthal, 1979; Rozeboom, 1960). More recently, some prominent methodologists have decried the practice as "terribly wrong," "an exceptionally bad idea" (Ziliak \& McCloskey, 2008, p. 2), though others, even more prominent, have been more tolerant so 
long as the procedure is used judiciously (e.g., Wilkinson, 1999). Some have suggested that we replace NHST completely with confidence intervals (Cumming, 2011; Cumming \& Finch, 2005; though see Morey, Hoekstra, Rouder, Lee, \& Wagenmakers, 2015), while others have proposed overthrowing the entire frequentist regime in favour of Bayesian analysis (Edwards, Lindman, \& Savage, 1963; Etz \& Vandekerckhove, 2017; Morey, Romeijn, \& Rouder, 2016; Wagenmakers, 2007).

Despite the various difficulties facing NHST today, few would expect the correct reporting of $p$-values in published reports of psychological research to pose a serious problem. Whatever challenges statistical analysis might present, the simple act of mechanically transferring the values of significance tests and their associated $p$-values from computer output to manuscript page would seem to be trivially easy. Recent research, however, has suggested that there are far more errors even in this basic aspect of statistical reporting than one might suppose.

During the early 2010s, a team of psychologists at the University of Amsterdam developed a computer program called "Statcheck" that has the ability sweep through large swathes of the published psychological research literature - tens of the thousands of articles - and detect instances where the reported $p$-value appears to not correctly correspond to the value of reported statistic: $\chi^{2}, F, r, t$, or $z$ (Nuijten, 2015). Using a sample of highly respected journals that cut across the major sub-disciplines of the field, ${ }^{1}$ covering

\footnotetext{
${ }^{1}$ Journal of Applied Psychology, Journal of Consulting and Clinical Psychology,
} Developmental Psychology, Journal of Experimental Psychology: General, Journal of 
the years from 1985 to 2013 , they found that (a) $54.4 \%$ of the more than 30,000 articles they examined contained instances of NHST that were detectable by Statcheck; (b) of the more than 16,000 articles with detectable NHSTs, virtually half (49.6\%) contained at least one inconsistency between a test statistic and its associated $p$-value; and (c) $12.9 \%$ of the NHST articles contained an inconsistency that would have, if corrected, required one to reverse one's decision about the statistical significance of the test result (Nuijten, Hartgernick, van Assen, Epskamp, \& Wicherts, 2016).

That virtually half of articles contain a $p$-value error will sound shocking, even unbelievable, to most psychological researchers. Because it was shocking, it quickly became the "headline number" of Nuijten et al.'s study. It is important to note, however, that, because this result was framed in terms of the percentage of articles in which at least one error was detected, it tended to sensationalize the situation somewhat. When, instead, the results are framed in terms of the percentage of individual (NHST) tests that were reported rather than percentage of articles that contained an inconsistent test, the numbers were not quite so alarming. Overall, (d) the percentage of $p$-values that were inconsistent with the corresponding test statistic was $9.7 \%$, and (e) the percentage of $p$ values that were so inconsistent they would have forced a reversal of the significance decision was just 1.4\% (Nuijten et al., 2016). One in ten $p$-values being incorrectly reported is certainly worthy of attention, but it offers a somewhat less apocalyptic vision

Personality and Social Psychology, Psychological Science, PLoS (where subject="psychology"), and Frontiers in Psychology. 
of the "headline number" that has been most often reported: that virtually half of published psychology articles are statistically erroneous.

Nuijten et al. (2016) reported that variations in the formats in which statistics are reported sometimes caused the program reading them incorrectly. For instance, Statcheck was unable to read the signs "<", ">", and "=", when publishers used image files (e.g., .gif) for these instead of ascii text characters. Statcheck was typically unable to read statistics that were reported in tabular form, rather than in-line in the text. Statcheck was only able to correctly read statistical reports when their formats exactly matched that specified in the Publication Manual of the American Psychological Association (2010). Thus, when writers strayed from those specifications, even marginally, Statcheck would fail to read them or report them as non-significant. Such unreadable versions included such common variants as, e.g., failing to put a space between the test statistic and the degrees of freedom $[t(37)=-4.93, p<.001]$, inserting sample sizes into the report $\left[\chi^{2}(1, N=226)=\right.$ $6.90, p<.01]$, inserting other statistics into the report $[F(2,70)=4.48, \mathrm{MSe}=6.61, p<$ $.02]$, and combining two results in a single sentence $[F(1,15)=19.9$ and $5.16, p<.001$ and $p<.05$, respectively] (examples taken directly from Nuijten et al., 2016, pp. 1206-1207). Even with these limitations, the information Statcheck was able to glean was well worth having; a first important step in checking the quality of the critical statistical information contained in the vast majority scientific research reports published in psychological journals. Beyond the figures that Nuijten et al. reported, however, we wondered whether the error rates were similar in the two major Canadian journals of 
psychological research, Canadian Journal of Experimental Psychology (just Canadian Journal of Psychology until 1990; hereafter referred to as CJEP) and Canadian Journal of Behavioral Science (hereafter CJBS). We decided to use Statcheck to test the articles published in these two journals over a 30-year period.

\section{Method}

We collected every article that appeared in the CJEP and CJBS between 1985 and 2014. Digital versions of the articles were available from our local university library. We removed from consideration all items that were not research articles - e.g., editorials, comments, reviews, obituaries.

Statcheck can, in principle, read articles in both PDF and HTML formats. We had originally intended to use the PDF versions that are available from PsycArticles. For reasons that are not clear, though, Statcheck was unable to reliably read many of the PDF documents that were available to us, especially those from early in the 30-year run of journals that we used. There may have been an incompatibility between Statcheck and the version of PDF in which these earlier articles had been preserved. In any case, one of the authors of Statcheck, Michèle Nuijten recommended that we use the HTML versions (personal communication, 2016).

We found that the HTML versions of these article had many typographical errors in them. These were, in all likelihood, the result of the process by which the original printed versions of the articles had been converted to digital form by means of optical character recognition (OCR) software. Although OCR technology has improved dramatically in recent 
years, early versions of OCR software often resulted in a large number of typographical errors, particularly if the printed font was an older style, or if the pages had even slightly foxed (browned) with age, as was common before the widespread introduction of acidfree paper in the late 1980 s. $^{2}$

Although, in the past, most OCR was followed up by careful hand editing to correct the errors introduced by it, the process by which a printed document is converted to PDF generates what is sometimes called "dirty OCR in the background": unedited ascii text that was never intended to be seen by the reader, but which can be used for (imperfect) digital searching of the document for particular strings of characters. (The photographic "page image" that most people associate with a PDF document cannot be digitally searched directly, only the ascii that lies behind it). This "dirty OCR" may have been the basis of the HTML versions that are now available online. Alternatively, the HTMLs may have been produced anew from printed documents through an OCR process that produced a new range of errors, and the sheer size of the conversion project (multiple volumes of multiple journals) precluded the practicability of substantial hand-editing.

In any case, our preliminary use of Statcheck on the HTMLs of the CJEP and CJBS articles, which is detailed below, led to the realization that many of the "errors" that

\footnotetext{
${ }^{2}$ The first author of the present article created the Classics in the History of Psychology website (http://psychclassics.yorku.ca), starting in the mid-1990s. He has scanned and converted via OCR thousands of pages of psychological publications. As such, he was very familiar with the problems typical of using OCR to convert older printed text.
} 
Statcheck found were not the result of statistical mistakes made by the authors of the articles but were, instead, errors that had been introduced somewhere during the process of converting the documents into digital versions. Hand-editing all 1838 articles was impracticable for us, so it was decided that we would sample the journal volumes from every fifth year - 1985, 1990, 1995, 2000, 2005, and 2010 - and hand-edit the statistical reports within those to the best of our ability. For instance, by visually inspecting the associated print versions, we ensured that all statistical test names and values were correct and readable. We ensured that spaces, parentheses, and decimal points were in the correct positions. We ensured that greater-than and lesser-than signs were rendered in ascii characters (rather than image files) and that they were in the correct orientations (as mentioned above, "<" and ">" were sometimes critically reversed by the conversion process).

In addition to correcting simple OCR errors in this sample, we also rendered statistical reports that Statcheck had been unable to read into readable versions. For instance, we removed insertions pertaining to sample size and MSe. We separated out significance tests that had been reported in combined form. We also converted statistics in French articles, which use commas in place of decimal points and semi-colons in place of commas, to the English-language standard that Statcheck presumes. We made an effort to convert statistics that had been reported in unreadable tabular form to a form readable by Statcheck. We found that the statistical information presented in tables was, unfortunately, often not sufficient to render it readable by Statcheck. Exact test values 
were often not reported, just levels of significance. Degrees of freedom values were frequently absent as well.

\section{Results}

\section{Full Population of Journal Articles}

The total number of articles used was 1839,887 from CJEP (med $=30.5 /$ year, SIQR = 3.38) and 952 from CJBS (med = 32/year, SIQR = 2.5). There was a downward trend in the number of CJEP articles published, from the mid-30s per year in the late 1980 s to the mid-20s per year in the early 2010s $(r=-.567, d f=885, p=.001)$. There was no significant trend over time for CJBS ( $r=-.038, d f=950, N S)$, but there was one anomalously high year for CJBS: 53 articles in 2014 (21 articles were published in issue no. 2 and 16 in issue no. 3, rather than the customary 7 or 8 ). When this one outlier was Winsorized to 36 (the next highest value), the magnitude of the negative correlation over time rose markedly, but did not quite attain significance $(r=-.322, d f=949, p=.08)$.

Of these 1839 articles, 909 contained Null Hypothesis Significance Tests (NHSTs) that could be detected by Statcheck. This represented $49.4 \%$ of the articles, a figure that is comparable to the $54.4 \%$ found by Nuijten et al. (2016). Of these 909 articles containing NHSTs, Statcheck was able to detect at least one error in 402 of them. This represents $44.2 \%$ of the NHST articles, which is also comparable to the $49.6 \%$ found by Nuijten et al. (2016).

As noted before, this way of expressing the finding - "nearly half of articles had at least one basic statistical error" - makes for a good headline but it also sensationalizes the 
problem. Thus, we recompiled our data based on the total number of tests (rather than the number of articles). Across all of the articles, Statcheck was able to detect 7840 NHSTs. Of these, $13.1 \%$ (1029) were declared to be "errors" by Statcheck. This is somewhat higher than the rate of $9.7 \%$ found by Nuijten et al. (2016), but not markedly so. The vast majority of these errors were minor (e.g., due to sloppy rounding) and, thus, would not have affected decisions about whether the findings were significant or not. Only 3.3\% (258) of the "errors" Statcheck found were so large that they would have reversed the significance decision, if corrected. This is still a little more than double the rate of $1.4 \%$ found by Nuijten et al. (2016).

The basic distribution of test statistics used in the articles was interesting as well: $67.8 \%$ of the tests were $F s, 18.3 \%$ were $t s, 8.2 \%$ were $\chi^{2} s, 2.9 \%$ were $r$ (not a test statistic, of course, but handled separately by Statcheck because so many researchers simply attach a $p$-values directly to $r$ s without reporting the intervening $t$-value), and $2.8 \%$ were zs. Statcheck also outputs an estimate of the proportion of NHSTs that were present but that it is was unable to interpret. This number is termed the "APA factor." For our data, the median APA factor was .78. That is, in general, Statcheck was able to assess $78 \%$ of the NHSTs that it recognized across all of the articles. (This estimate relies on Statcheck being able to determine that a statistical report was presented, but that it was unable to read it. There were many other statistical reports that Statcheck was unable to detect at all.) 


\section{Corrected Sample of Journal Articles}

As described in the Method section, we decided to sample every fifth year of the journals and correct them by hand. It is not certain, however, that our sample would have exactly the same values with respect to the accuracy of the NHSTs as the complete population had. Thus, to ensure that the comparisons would be valid, we recalculated the same statistics as we had for the full population of articles on just the sample but prior to introducing our corrections.

The sample consisted of 368 articles (20.0\% of population). Of those, 174 articles contained detectable NHST tests: (47.3\%, slightly below the population value of $49.4 \%$ ). Statcheck detected at least one "error" in 69 of those articles (39.7\%, somewhat lower than the population value of $44.2 \%)$. Out of a total of 1398 detectable tests in the sample (17.8\% of the detectable tests in the population), Statcheck detected 161 errors $(11.5 \%$, somewhat below the population value of $13.1 \%)$. The number of errors that would have reversed the decision about significance was 29 (2.1\%, somewhat below population value of $3.3 \%$, and closer to the value of $1.4 \%$ found by Nuijten et al., 2016). 


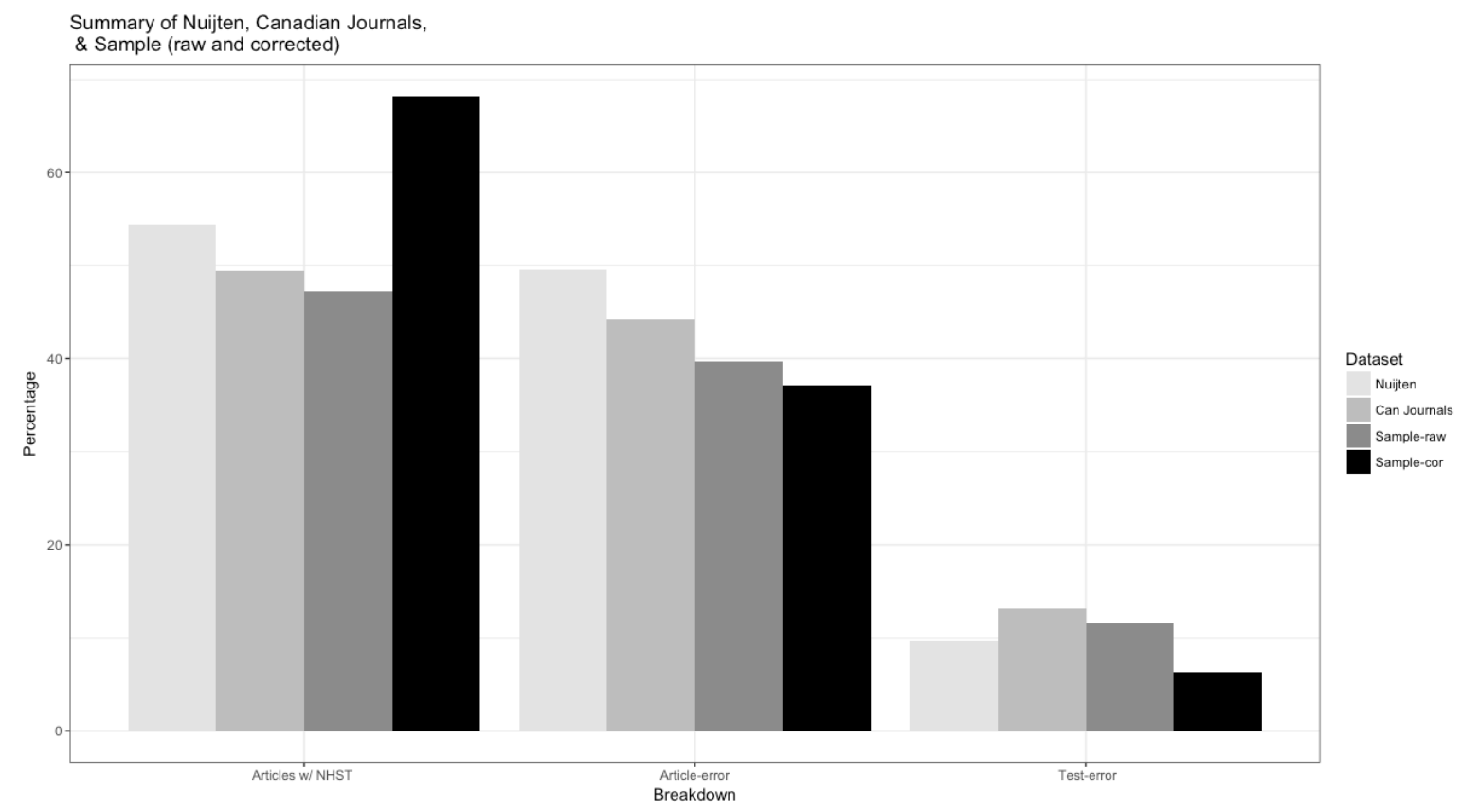

FIGURE 1

After the sample had been corrected by hand, some of these numbers shifted markedly. Of the 368 articles in the sample, Statcheck was, after correction, able to detect 251 that contained NHSTs $(68.2 \%$, or $44 \%$ more articles compared to the uncorrected sample). Statcheck detected errors in 93 of these articles (37.1\%, slightly lower than the $39.7 \%$ we found in the uncorrected sample). Statcheck was able to detect 2726 NHSTs, a sizeable increase of $95 \%$ compared to the uncorrected sample. Among those corrected tests, it now found 171 errors $(6.3 \%$, or $45 \%$ fewer errors than in the uncorrected sample). The number of errors that would have reversed the decision about significance was now 73 (2.6\%, slightly more than in the uncorrected sample).

In short, the hand correction greatly increased the number of articles that were found to contain NHSTs and it very nearly doubled the number of individual tests that 
were detected. The hand correction only slightly affected the percentage of articles with at least one error, but it reduced by nearly one-half the rate of errors found among the tests themselves. The rate at which those errors would have reversed significance decisions was left nearly unaffected.

Using the corrected sample, the two journals were compared to each other to see whether errors tended to occur more in the perception/cognitive side or in the social/personality of the discipline. The two were virtually identical in the percentage of articles with at least one error (CJEP: 45/124 = 36.3\%, CJBS: 45/128 = 35.2\%). A slightly higher rate of erroneous tests was found in CJBS $(84 / 1188=7.1 \%)$ than were found in $\operatorname{CJEP}(81 / 1541=5.3 \%)$.

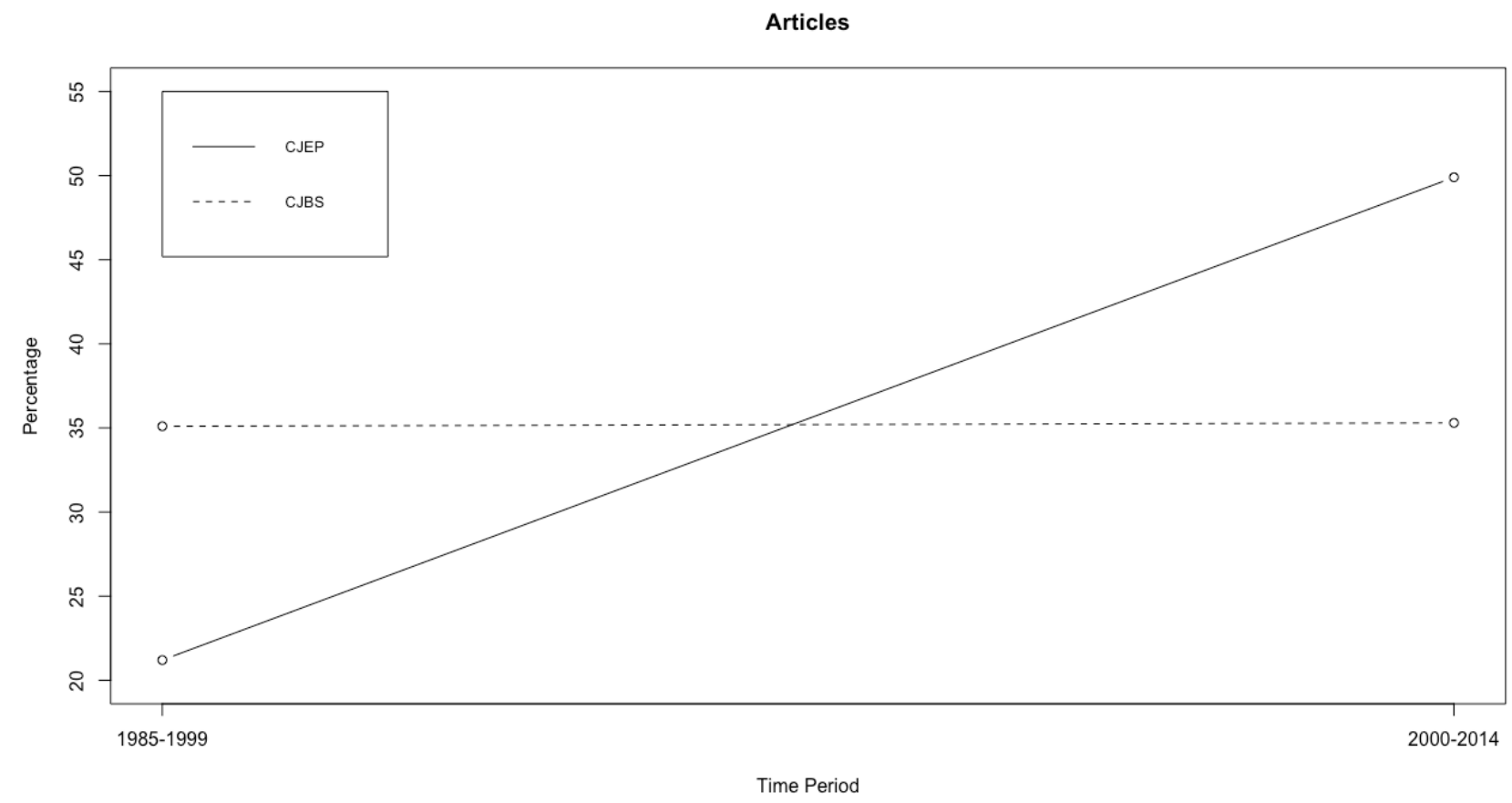

FIGURE 2 
To see whether errors had increased or decreased over time, the 30-year time period was divided in half (1985-1999 vs. 2000-2014). A markedly smaller percentage of articles were found to contain errors in the earlier journals $(39 / 134=29.1 \%)$, than in the later journals $(51 / 118=43.2 \%)$. When these numbers were broken down by journal, however, it became clear that the increase was entirely attributable to the CJEP articles, in which the error rate rose from $21.1 \%$ of the earlier articles to $49.3 \%$ of the later articles. The CJBS articles, by contrast, remained virtually flat across the two time periods (early: 35.1\%; late: $35.3 \%)$. Interestingly, however, this difference between the two journals was seen only when looking for the number of articles with at least one error. When one looked instead at the rate of individual tests with errors, the difference between the early (5.5\%) and late (6.5\%) time periods was minimal. Separating the two journals, the rate of erroneous tests was slightly higher in CJBS (early: 6.4\%; late: $8.2 \%$ ) than in CJEP (early: $4.2 \%$; late: $5.8 \%$ ) in both time periods. 


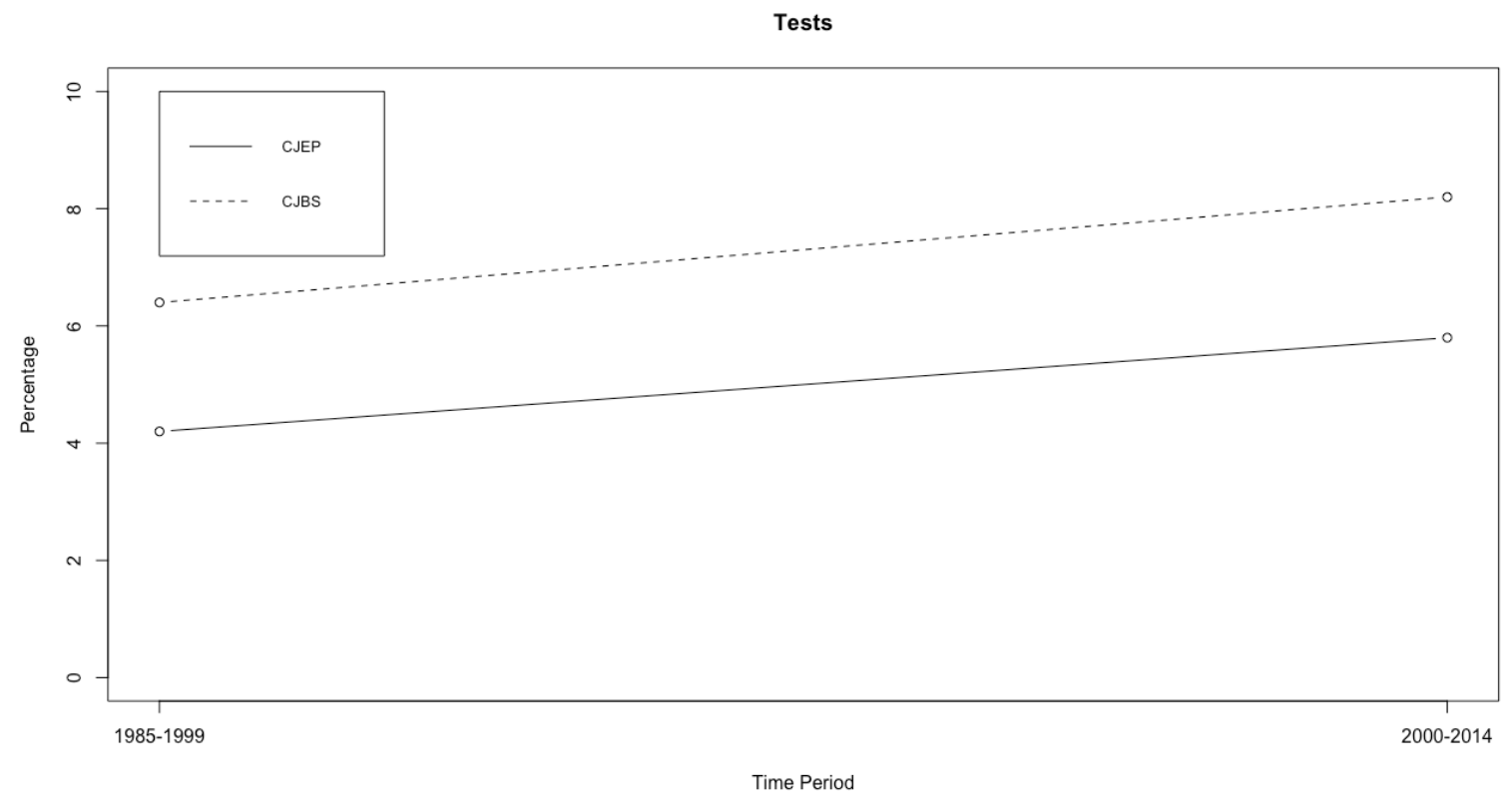

FIGURE 3

\section{Discussion}

The present study set out to discover whether the rates of erroneous $p$-values in Canadian psychology journals were comparable to those found by Nuijten et al. (2016), using Statcheck, in major American and European psychology journals. Examining 30 years of CJEP and CJBS articles, we found that the nominal error rates were approximately the same: about half of articles contained at least one apparent error and about $10 \%$ of the tests themselves were erroneous. However, we also found many typographical errors in the electronic versions of the articles, probably introduced in the process of converting printed articles into PDFs. So, we hand-corrected a sample of $20 \%$ of the articles, spread evenly throughout the time period. 
After making these corrections, we found an increase of nearly $50 \%$ in the number of articles where Statcheck could detect at least one NHST, and a near-doubling of the number of individual NHSTs. The rate of erroneous tests was almost halved, showing that the original rates had reflected nearly as many typographical errors in the electronic versions of the articles as actual statistical errors in the published articles. Interestingly, though, despite the sharp drop in the rate of test errors, the proportion of articles with at least one NHST error decreased only slightly.

We also investigated whether the journals had different rates of NHST errors, as well as whether there had been a change in error rates over time. We found that the rate of articles with at least one error had risen between the early and late time periods, from about $30 \%$ to somewhat above $40 \%$. Breaking the data down by journals, though, showed that the rise was attributable to CJEP, which leapt from an article error rate of about $20 \%$ in the earlier half of the time period to a rate of nearly $50 \%$ in the latter half. This difference was not found, however, when looking at the rate of errors among tests themselves. CJBS had a somewhat higher rate of erroneous tests in both the early and late time periods.

Perhaps the most important thing we found was that typographical errors seem to have been frequently introduced in the process of converting articles from print to electronic form. Because of these errors, Statcheck seems to be unable to detect many instances of NHSTs. The number of tests Statcheck could detect nearly doubled when we corrected the sample of electronic articles by hand. Moreover, where Statcheck does 
detect instance of NHSTs, it may be overestimating the problem of incorrect $p$-values because often those incorrect values were not incorrect in the original article, only in the converted electronic version.

Other programs have recently been developed for checking the accuracy of the statistics reported. GRIM (for "granularity-related inconsistency of means") checks whether the arithmetic means reported are actually possible, given the sample size (Heathers, 2016; see Bartlett, 2017 for a salient application). SPRITE (for "sample parameter reconstruction via iterative techniques") is another, by the same researcher, which uses the reported sample size, mean, and standard deviation to investigate the character possible data distributions that could have produced them (Heathers, 2017). This is not solely a psychological phenomenon. Jennifer Byrne and Cyril Labbé have developed a program called "Seek \& Blastn" to detect incorrectly identified nucleotide fragments in published cancer research (Phillips, 2017). All efforts of these sorts, useful as they are in principle, will have to take care that the programs are reading the text correctly prior to declaring to have found an error.

Nevertheless, even if it is true that Statcheck is overestimating the problem of incorrect $p$-values due to typographical errors, the type of error it seeks should be virtually non-existent in the published literature. Professional psychological researchers are mostly quite well versed in the use of NHST. For the past few decades now, most statistical calculations have been handled by computer, so there should be vanishingly few computational errors. (One reviewer suggested that the problem might actually lie in 
researchers misinterpretations of the (sometimes copious) output that their statistical packages produce.) We would expect researchers to be conscientious enough to faithfully copy numerical values from computer printout to article draft. Nevertheless, more errors than one would expect appear to still be present in the published literature. Perhaps the journal editorial process should change to include a stage in which statistical reports are explicitly examined by a reviewer or editor to ensure that they are coherent and correct. 
References

American Psychological Association. (2010). Publication manual of the American

Psychological Association (6th ed.). Washington DC: American Psychological Association.

Antonakis, J. (2017). On doing better science: From thrill of discovery to policy implications. The Leadership Quarterly, 28(1), 5-21. https://doi.org/10.1016/j.leaqua.2017.01.006

Bakan, D. (1967). The test of significance in psychological research. In On Method (p. Chapter 1). San Francisco: Jossey-Bass.

Bartlett, T. (2017, March 17). Spoiled Science: How a seemingly innocent blog post led to serious doubts about Cornell's famous food laboratory. The Chronicle of Higher Education. Retrieved from http://www.chronicle.com/article/SpoiledScience/239529

Carpenter, S. (2012, September 6). Harvard Psychology Researcher Committed Fraud, U.S. Investigation Concludes. Retrieved October 10, 2017, from http://www.sciencemag.org/news/2012/09/harvard-psychology-researchercommitted-fraud-us-investigation-concludes

Carroll, A. E. (2017, May 29). Science Needs a Solution for the Temptation of Positive Results. The New York Times. Retrieved from https://www.nytimes.com/2017/05/29/upshot/science-needs-a-solution-for-thetemptation-of-positive-results.html 
Cohen, J. (1962). The statistical power of abnormal-social psychological research: a review. Journal of Abnormal and Social Psychology, 65, 145-153.

Cohen, J. (1992). A power primer. Psychological Bulletin, 112, 155-159.

Cohen, J. (1994). The earth is round ( $p<.05)$. American Psychologist, 49, 997-1003.

Cumming, G. (2011). Understanding the new statistics: Effect sizes, confidence intervals, and meta-analysis. London, UK: Routledge.

Cumming, G., \& Finch, S. (2005). Inference by eye: Confidence intervals and how to read pictures of data. American Psychologist, 60, 170-180. https://doi.org/10.1037/0003-066X.60.2.170

Edwards, W., Lindman, H., \& Savage, L. J. (1963). Bayesian statistical inference for psychological research. Psychological Review, 70, 193-242.

Enserink, M. (2012, November 28). Final Report: Stapel Affair Points to Bigger Problems in Social Psychology. Retrieved October 10, 2017, from http://www.sciencemag.org/news/2012/11/final-report-stapel-affair-pointsbigger-problems-social-psychology

Etz, A., \& Vandekerckhove, J. (2017). Introduction to Bayesian Inference for Psychology. Psychonomic Bulletin \& Review, 24, 1-30. https://doi.org/10.3758/s13423-0171262-3

Gigerenzer, G. (2004). Mindless statistics. Journal of Socio-Economics, 33, 587-606. Heathers, J. (2016, May 23). The GRIM test - a method for evaluating published research. Medium [blog]. Retrieved November 24, 2017, from 
https://medium.com/@jamesheathers/the-grim-test-a-method-for-evaluatingpublished-research-9a4e5f05e870

Heathers, J. (2017, March 3). Introducing SPRITE (and the Case of the Carthorse Child). Hackernoon [blog]. Retrieved November 24, 2017, from https://hackernoon.com/introducing-sprite-and-the-case-of-the-carthorse-child$58683 c 2 b f e b$

John, L. K., Loewenstein, G., \& Prelec, D. (2012). Measuring the Prevalence of Questionable Research Practices With Incentives for Truth Telling. Psychological Science, 23, 524-532.

Kessler, A. (2017, August 13). Studies Are Usually Bunk, Study Shows. Wall Street Journal. Retrieved from https://www.wsj.com/articles/studies-are-usually-bunk-studyshows-1502660991

Lykken, D. T. (1968). Statistical significance in psychological research. Psychological Bulletin, 70, 151-159.

Masicampo, E. J., \& Lalande, D. R. (2012). A peculiar prevalence of p values just below .05. Quarterly Journal of Experimental Psychology, 65, 2271-2279.

Meehl, P. E. (1967). Theory testing in psychology and physics: A methodological paradox. Philosophy of Science, 34, 103-115.

Meehl, P. E. (1978). Theoretical risks and tabular asterisks: Sir Karl, Sir Ronald, and the slow progress of soft psychology. Consulting and Clinical Psychology, 46, 806-834. 
Morey, R. D., Hoekstra, R., Rouder, J. N., Lee, M. D., \& Wagenmakers, E.-J. (2015). The fallacy of placing confidence in confidence intervals. Psychonomic Bulletin \& Review, 23, 103-123. https://doi.org/10.3758/s13423-015-0947-8

Morey, R. D., Romeijn, J., \& Rouder, J. N. (2016). The philosophy of Bayes factors and the quantification of statistical evidence. Journal of Mathematical Psychology, 72, 618. https://doi.org/10.1016/j.jmp.2015.11.001

Nuijten, M. B. (2015, November 17). Making error detection easier - and more automated: A guest post from the co-developer of "statcheck." Retrieved April 7, 2017, from http://retractionwatch.com/2015/11/17/making-it-easier-and-moreautomated-to-find-errors-a-guest-post-from-the-co-developer-of-statcheck/

Nuijten, M. B., Hartgernick, C. H. J., van Assen, M. A. L. M., Epskamp, S., \& Wicherts, J. M. (2016). The prevalence of statistical reporting errors in psychology (1985-2013). Behavioral Research Methods, 48, 1205-1226.

Nunnally, J. C. (1960). The place of statistics in psychology. Educational and Psychological Measurement, 20, 641-650.

Open Science Collaboration. (2015). Estimating the reproducibility of psychological science. Science, 349(6251). https://doi.org/10.1126/science.aac4716

Phillips, N. (2017). Online software spots genetic errors in cancer papers. Nature News, 551(7681), 422. https://doi.org/10.1038/nature.2017.23003

Rosenthal, R. (1979). The "file drawer problem" and tolerance for null results. Psychological Bulletin, 86, 638-641. https://doi.org/10.1037/0033-2909.86 
Rozeboom, W. W. (1960). The fallacy of the null hypothesis significance test. Psychological Bulletin, 57, 416-428.

Simmons, J. P., Nelson, L. D., \& Simonsohn, U. (2011). False-positive psychology: Undisclosed flexibility in data collection and analysis allows presenting anything as significant. Psychological Science, 22(11), 1359-1366.

https://doi.org/10.1177/0956797611417632

Wagenmakers, E.-J. (2007). A practical solution to the pervasive problems of $p$ values. Psychonomic Bulletin \& Review, 14, 779-804.

Wilkinson, L. (1999). Statistical methods in psychology journals: Guidelines and explanations. American Psychologist, 54(8), 594-604.

https://doi.org/10.1037/0003-066X.54.8.594

Yong, E. (2015, August 27). How Reliable Are Psychology Studies? The Atlantic. Retrieved from https://www.theatlantic.com/science/archive/2015/08/psychology-studiesreliability-reproducability-nosek/402466/

Ziliak, S. T., \& McCloskey, D. N. (2008). The Cult of Statistical Significance. Ann Arbor, MI: University of Michigan Press. Retrieved from https://www.press.umich.edu/186351/cult_of_statistical_significance 
Figures Captions

Figure 1. Grouped bar graphs comparing the findings of Nuijten et al. (2016), our full population of Canadian journals, our uncorrected sample of Canadian journals, and our corrected sample of Canadian journals.

Figure 2. Line graphs comparing the rates of article errors in CJEP and CJBS, across the earlier and latter halves of the time period. Note that CJEP rose over time while CBJS remained essentially stable.

Figure 3. Line graphs comparing rates of test errors in CJEP and CJBS, across the earlier and letter halves of the time period. Note that there is only a slight rise in each journal over time, and that CJBS has a somewhat higher test error rate than CJEP in both time periods. 